\title{
A Model of Signaling for Establishing of LSPs for Multicast Communication over GMPLS Networks
}

\author{
Rafael P. Esteves ${ }^{1}$, Antônio J. G. Abelém ${ }^{1}$, Ewerton $\mathrm{Vaz}^{2}$ and Michael A. \\ Stanton ${ }^{3}$ \\ 1 Federal University of Pará, Department of Informatics \\ Rua Augusto Corrêa, 01, 66075-110, Belém, Pará, Brazil \\ \{esteves, abelem\}@ufpa.br \\ WWW home page: http://www.cultura.ufpa.br/abelem \\ 2 Federal University of Pará, Computer Science Graduate Program \\ Rua Augusto Corrêa, 01, 66075-110, Belém, Pará, Brazil \\ ewerton@ufpa.br \\ WWW home page: http://www.ufpa.br/ppgcc \\ 3 Fluminense Federal University, Institute of Computing \\ Rua Passo da Pátria, 156, bloco E, sala 350, 24210-240, Niterói, RJ, Brazil \\ michael@ic.uff.br \\ WWW home page: http://www.ic.uff.br/-michael/
}

\begin{abstract}
Label switching, which in IP networks is exemplified by MPLS and its extensions MPLambdaS and GMPLS, appears as one of the best alternatives to offer a reliable and flexible control plane for WDM networks, since it allows the integration of the IP Protocol with WDM technology, when lambdas are associated with labels, implements powerful traffic-engineering mechanisms, and provides several alternative schemes for fault-tolerance, as well as support for quality of service (QoS). However, almost all the definitions and standardizations for MPLS are restricted to unicast communication, leaving support for multicast communication for future work. In the specific case of the triggering problem for LSPs (Label Switched Paths), there is still no consensus about the best strategy for multicast communication. This paper proposes an algorithm for traffic-driven triggering of LSPs, based on MFCs (Multicast Forwarding Caches), and has the advantage of being a schema which is compatible with several multicast routing protocols. To validate the proposed algorithm we carry out simulation studies using the NS-2 (Network Simulator) simulation platform.
\end{abstract}

Please use the following format when citing this chapter:

Esteves, R.P., Abelém, A.J.G., Vaz, E., Stanton, M.A., 2006, in IFIP International Federation for Information Processing, Volume 213, Network Control and Engineering for QoS, Security, and Mobility, V, ed. Gaïti, D., (Boston: Springer), pp. 171-186. 


\section{Introduction}

The increasing demand for sophisticated network applications, allied to the growth of the Internet traffic, has lead to great efforts in the search of improvements in data transmission technologies with the intention of satisfying the increasing demand for bandwidth.

So far as optical networking is concerned, WDM (Wavelength Division Multiplexing) appears as the main advance in the transmission area, because it allows transmission rates near to the theoretical limit of optical fibers, of the order of dozens of terabits a second [1]. An essential issue in optical network design is defining how the network will be controlled, that is, what type of signalling will be responsible for resource reservation, route determination and fault handling, among other functions that constitute the control plane.

Label switching, which in IP networks is exemplified by MPLS (Multiprotocol Label Switching) [2], was extended through GMPLS (Generalized Multiprotocol Label Switching) [3] to operate with several different network technologies, where the label can be represented in other ways, for example, as time-slots in TDM networks, as physical switch ports and as wavelengths (lambdas) in WDM networks.

GMPLS appears as one of the best alternatives to offer a reliable and flexible control plane for WDM networks, since it allows the integration of the IP Protocol with WDM technology, when lambdas are associated with labels, implements powerful traffic-engineering mechanisms, and provides several alternative schemes for fault-tolerance, as well as support for quality of service (QoS). However, almost all the definitions and standardizations for MPLS are restricted to unicast communication, leaving support for multicast communication for future work.

In multicast based on label switching, the establishment of a label-switched path (LSP) can be made in three ways [4]: request-driven, topology-driven or trafficdriven. However, there is no consensus on which is the best alternative, since all three present pros and cons. In a similar way, controversies exist about the type of control to be adopted in multicast communication (independent or ordered) and about the method for distributing labels for a certain equivalence class, which can either be initiated by the egress LSR of the link, using the on-demand or unsolicited approaches, or by the ingress LSR of the link, using the on-demand, unsolicited or implicit approaches [4]. Thus we may conclude that a great opportunity is presented to analyze and reformulate some aspects of multicast communication and its use in a label switching context.

The objective of this paper is to propose an algorithm for the traffic-driven triggering of LSPs based on MFCs (Multicast Forwarding Caches), which are structures derived from the multicast routing tables found in the implementations of IP multicast (DVMRP, PIM), with the purpose of obtaining a scheme which is compatible with several implementations of multicast routing protocols. Basically, in this approach, when a node needs to create an entry in an MFC to store information about multicast groups, an LSP request is sent and processed by all the nodes in the direction of the source node. To validate the proposal, simulations were carried out using the NS-2 (Network Simulator) simulation platform. 
As our proposal is related to label-switching in a general way, it can be associated, in addition to MPLS, to the improvements developed to bring label switching to the next-generation networks, such as GMPLS.

In addition to this introductory section, the paper is organized in more 4 sections. Section 2 discusses the characteristics of existing LSP triggers, presented in the literature. The algorithm for establishing LSPs is described in Section 3. Section 4 presents the simulation experiments used to validate the proposed algorithm. Section 5 presents conclusions and proposals for future work.

\section{Triggering Multicast LSPs}

One of the main discussion points concerning the use of label switching for multicast communication is the choice of method to be used to establish the label-switched paths.

Ooms et al. [4] define three approaches to this task: request-driven, topologydriven and traffic-driven. In the request-driven approach the label advertisement messages are encapsulated in control messages of the multicast routing protocol, such as Join messages, or in RSVP messages. This approach takes advantage of the characteristics of existing routing protocols, but can require that such protocols include explicit control messages, such as in PIM-SM, restricting its applicability to this class of protocol.

The encapsulation of label advertisements in native control messages of the multicast routing protocol is usually referred as "piggy-backing". This method has some problems, such as the fact that it excludes dense-mode protocols, since these do not use explicit control messages, and the modifications needed to add these messages to the protocol would be very expensive. Piggy-backing also makes impractible the use of other approaches to LSP triggering, as well as requiring extensions to the routing protocol to handle other LDP (Label Distributon Protocol) functions, such as peer discovery, and contributing to an increase in control traffic.

The topology-driven approach maps a level 3 multicast tree to a level 2 tree. The disadvantage of this is that labels are consumed even if there is no traffic for a certain multicast tree.

Traffic-driven LSP triggering has the advantage of consuming less labels than the other methods, because LSPs will only be established if there is demand for traffic. To achieve this, the technique monitors data structures known as MFCs (Multicast Forwarding Caches). MFCs are subsets of multicast routing tables that store information relating only to multicast groups that handle traffic [5]. If no entry exists in the MFC for a given multicast packet, one will be created. If the routes change afterwards, the MFC will be updated.

MFCs are used in most IP multicast implementations in UNIX systems and, as they are kept in the kernel of the operating system, traffic-driven triggering can be used with several different multicast routing protocols, making this approach more attractive than the alternatives.

Although these different approaches have all been proposed, a detailed definition of their mechanisms is needed, and some of them are not compatible with all existing 
multicast routing protocols. In this paper we describe an algorithm to establish LSPS based on traffic-driven triggering, since this approach has the advantage of being compatible with several multicast routing protocols and also consumes fewer labels than other multicast LSP triggers.

\section{An Algorithm for LSP Establishment for Multicast Communication Based on MFCs}

\subsection{Reference Model}

The reference model used in this article consists of IP/MPLS (we will use the MPLS term to refer to MPLS and its extensions MPAS and GMPLS) routers connected by means of an optical internetwork, using dynamically switched lightpaths (Fig. 1). The optical networks composing this internetwork are based on OCS (Optical Circuit Switching) [6] and MPLS paradigms. The choice of OCS is due to its low complexity, and, consequently, low implementation cost compared with other optical switching paradigms. On the other hand, the choice of MPLS, as pointed out in Section II, is justified by its flexibility and support for traffic engineering, as well as its being extensible to work with WDM technology, with the use of wavelengths as labels.

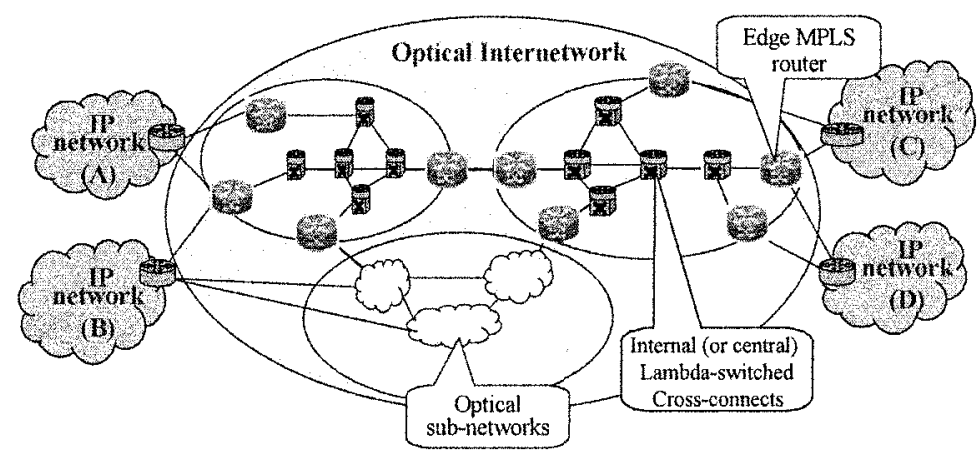

Fig. 1. Model network used in this proposal, composed of multiple optical switching devices (LSC) interconnected by an optical mesh.

We assume that the optical internetwork consists of a number of optical networks, each of which might be operated by a separate administrative entity. Each optical network can be made up of subnetworks comprised of a number of optical switching devices capable of label switching (Lambda Switching Crossconnects LSCs) and interconnected by some non-specific topology of optical links. For simplicity, we assume there exists a one-to-one mapping between WDM switches and their IP-addressable controllers.

Signaling in an optical internetwork is performed out of band, and uses just one (high capacity) signaling channel (or lambda) per fiber. Signaling messages are 
electronically processed by all nodes, including internal ones. Data packets are not processed at internal nodes, and we make no assumptions about the data transmission rate. Network intelligence is concentrated essentially at the network edge, and no global synchronization is required.

We further assume the use of the integrated control model, as presented in [7], where both the IP and optical domains are managed in a unified way, with a single routing protocol and a single control plane based on MPLS. In the case of the internetwork consisting of a single Autonomous System (AS), a single intradomain routing protocol will be considered. When several ASs are involved, an interdomain routing protocol will also have to be used. Both intra- and interdomain routing protocols need to include the necessary extensions for optical technologies.

We also consider that all devices run IP Multicast and that optical devices have full light-splitting capability. The equipment must support both level 2 (MPLS) and level 3 routing.

\subsection{Description of the proposed algorithm}

In this section we describe an algorithm for traffic-driven LSP triggering. Our purpose is to detail the actions needed to establish LSPs, using as few resources as possible. The algorithm works as follows:

Every time a node receives a multicast packet, it verifies if there is information about the destination in the multicast forwarding cache (MFC). If this information is not found this node requests the creation of an entry in this structure.

When a leaf node (a node that is a destination of the multicast data) needs to request an entry in the MFC it acquires a label (Label can be represented by a wavelength in a MP $\lambda \mathrm{S} / \mathrm{GMPLS}$ context) for itself and propagates to the previous node on the route from the source the following information: the label to be used and the FEC (Forwarding Equivalence Class) of the LSP that is being built.

This same information will be used to create entries in the LIB (Label Information Base) of the node. The LIB in each LSR (Label Switched Router) stores data that will later be used in label-processing. A LIB entry usually contains the following fields: FEC, incoming label, incoming interface, outgoing label and outgoing interface [8].

The label sent will be used by the previous node (following the traffic direction) on the label swapping operation, that is to change the incoming label value with the outgoing label value (the incoming label of the next hop towards the leaf-nodes).

An intermediate node will also assign a label, create a LIB entry and forward this information along the path to the previous node in a similar way.

The multicast traffic source node does not request labels because it gets them from the downstream nodes belonging to the multicast tree.

When an intermediate node needs to replicate packets, it will receive several labels for the same equivalence class from the outgoing interfaces of the multicast distribution tree. If this happens, the node creates the additional entries in the LIB with the same FEC and the same incoming label. Fig 2. illustrates the algorithm.

This upstream signaling approach was designed to guarantee that all the nodes along the path have labels for a given FEC. The FECs are associated with the traffic 
source in this approach, in other words, the packets that originate at a given node and belong to the same multicast group also belong to a single FEC. It is worth pointing out that in this approach a single FEC is associated with several LSPs that have a common source.

To initiate this LSP triggering process, traffic has to arrive at a leaf node belonging to a multicast group. This data forwarding demands level 3 routing, since there is still no LSP to route these packets at level 2, therefore, the devices used in this approach must support this functionality.

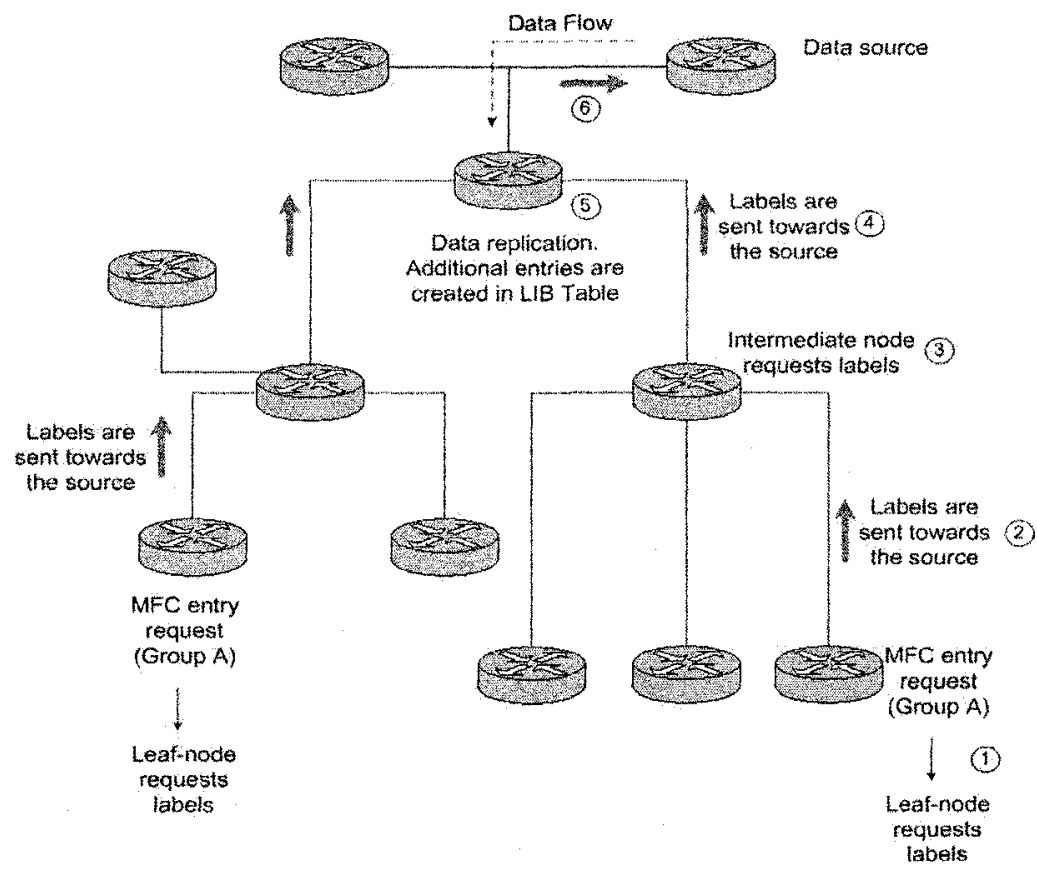

Fig. 2. Proposed algorithm for LSP triggering.

We now describe the format of the LIB entries created by the algorithm for each type of node (leaf, intermediate and source). On LSP triggering a LIB entry at the leaf node is created using the following format:

Table 1. LIB entry for a Leaf Node

\begin{tabular}{|l|l|l|l|l|}
\hline FEC & $\begin{array}{l}\text { Incoming } \\
\text { Interface }\end{array}$ & $\begin{array}{l}\text { Incoming } \\
\text { Label }\end{array}$ & $\begin{array}{l}\text { Outgoing } \\
\text { Interface }\end{array}$ & $\begin{array}{l}\text { Outgoing } \\
\text { Label }\end{array}$ \\
\hline $\begin{array}{l}\text { Source node } \\
\text { identifier }\end{array}$ & $\begin{array}{l}\text { Next upstream } \\
\text { node }\end{array}$ & $\begin{array}{l}\text { Requested at } \\
\text { this moment }\end{array}$ & -1 & -1 \\
\hline
\end{tabular}


$F E C$ : The forwarding equivalence class that is associated with the source node in this case.

Incoming Interface: Defines where the traffic that arrives to that node comes from, that is, the next upstream node in this case.

Incoming Label: The incoming label consists of the label that is assigned by this node. It is requested at this moment, because this parameter will be forwarded to the upstream node to guarantee the creation of a consistent LSP between the leaf node and the data source.

Outgoing Interface: Represents the next hop of the packets. Here the value -1 is used because this is a leaf node, and there is no downstream node.

Outgoing Label: It represents the new value of the label. Here the value -1 is defined because it is a leaf node.

As the signaling messages reach intermediate nodes, the one of more entries are created in each of their LIBs, using the following format:

Table 2. LIB entry for intermediate nodes

\begin{tabular}{|l|l|l|l|l|}
\hline FEC & $\begin{array}{l}\text { Incoming } \\
\text { Interface }\end{array}$ & $\begin{array}{l}\text { Incoming } \\
\text { Label }\end{array}$ & $\begin{array}{l}\text { Outgoing } \\
\text { Interface }\end{array}$ & $\begin{array}{l}\text { Outgoing } \\
\text { Label }\end{array}$ \\
\hline $\begin{array}{l}\text { Source node } \\
\text { identifier }\end{array}$ & Upstream node & $\begin{array}{l}\text { Requested at } \\
\text { this moment }\end{array}$ & $\begin{array}{l}\text { Next } \\
\text { downstream } \\
\text { node }\end{array}$ & $\begin{array}{l}\text { Incoming label } \\
\text { of the next } \\
\text { downstream } \\
\text { node }\end{array}$ \\
\hline
\end{tabular}

FEC, Incoming Interface and Incoming Label are interpreted and defined in a similar way to the leaf node.

Outgoing Interface: It is defined with the identifier of the next node towards a certain downstream leaf node, where an LSP was already triggered.

Outgoing Label: Defined with the value of the incoming label corresponding to the next downstream node in Outgoing Interface.

If this node is a data replicator, additional similar entries are created in the LIB with the same incoming interface and label but different outgoing interfaces and labels.

When the signaling message reaches the source node, one or more entries will be created in its LIB with the following format:

Table 3. LIB entry for the source node

\begin{tabular}{|l|l|l|l|l|}
\hline FEC & $\begin{array}{l}\text { Incoming } \\
\text { Interface }\end{array}$ & $\begin{array}{l}\text { Incoming } \\
\text { Label }\end{array}$ & $\begin{array}{l}\text { Outgoing } \\
\text { Interface }\end{array}$ & $\begin{array}{l}\text { Outgoing } \\
\text { Label }\end{array}$ \\
\hline $\begin{array}{l}\text { Source node } \\
\text { identifier }\end{array}$ & -1 & -1 & $\begin{array}{l}\text { Next } \\
\text { downstream } \\
\text { node }\end{array}$ & $\begin{array}{l}\text { Incoming label } \\
\text { of the next } \\
\text { downstream } \\
\text { node }\end{array}$ \\
\hline
\end{tabular}


FEC, Outgoing Interface and Outgoing Label are interpreted and defined as shown in Table 2.

Incoming Interface: Here the value -1 is defined since, because it is the source node, there is no further upstream node.

Incoming Label: Here the value -1 is defined because it is the source node.

\section{Validation and Analysis of the Proposed Mechanism}

\subsection{Tools Used}

In order to analyze the proposed signaling and to verify its applicability, simulations were carried out using the NS-2 (Network Simulator) platform. NS-2 [9] is a discrete event-oriented simulator for computer networks much used by the scientific community for analysis and validation of proposals, having as a main attraction an extensible architecture that can easily incorporate new functionality.

NS-2 has modules for label switching, MNS ("MPLS Network Simulator") [10], and for WDM optical networks simulations based in lambda (circuit) switching, OWNS ("Optical WDM Network Simulator") [11]. It also supports multicast communications through the implementation of dense-mode protocols (PIM-DM, DVMRP), sparse-mode protocols (PIM-SM) and shared trees.

OWNS is an extension module for NS-2 that allows simulation of WDM networks that use circuit switching, also known as lambda switching. It also has components as optical switching nodes, wavelength multiplexed links, and routing and wavelength assignment algorithms.

MNS adds MPLS simulation capabilities to NS-2, including some characteristics as label switching, support for LDP and CR-LDP protocols, flow aggregation and constraint-based routing, and it allows the use of some LSP protection and restoration techniques.

The version of NS-2 used in this study was $2.1 \mathrm{~b} 6$, because it has better compatibility with the NS- 2 extensions, OWNS and MNS, that are fundamental for the required simulation context.

However, these modules do not cooperate with each other, and they do not support multicast communication. Therefore, the simulation of the proposed LSP triggering mechanism demands the following preliminary conditions:

- The integration of OWNS and MNS modules, described in detail in [12] [13], which enables us to characterize the GMPLS architecture, where the labels are associated with wavelengths (lambdas). It is worth pointing out that GMPLS adds new functions to the existing ones in MPLS: however, in our case, the other characteristics of GMPLS were not implemented, as we just needed the mapping between labels and lambdas and the functionalities already defined in the MNS module.

- Modifications and adaptations to extend the previously developed combined WDM-MPLS structure, to add support for multicast communication, including the development of replicators that can handle labels and the implementation of the proposed algorithm. Boudani and Cousin [14] have proposed a simulation tool based on 
NS-2 that implements multicast communication in MPLS networks. However, this latter proposal is limited to the PIM-SM multicast routing protocol, which distributes labels through explicit signaling, using Join messages, while our proposal works with several protocols since it implements the traffic-driven LSP triggering.

Fig. 3 shows the NS-2 node structure developed to support multicast transport in networks with label switching.

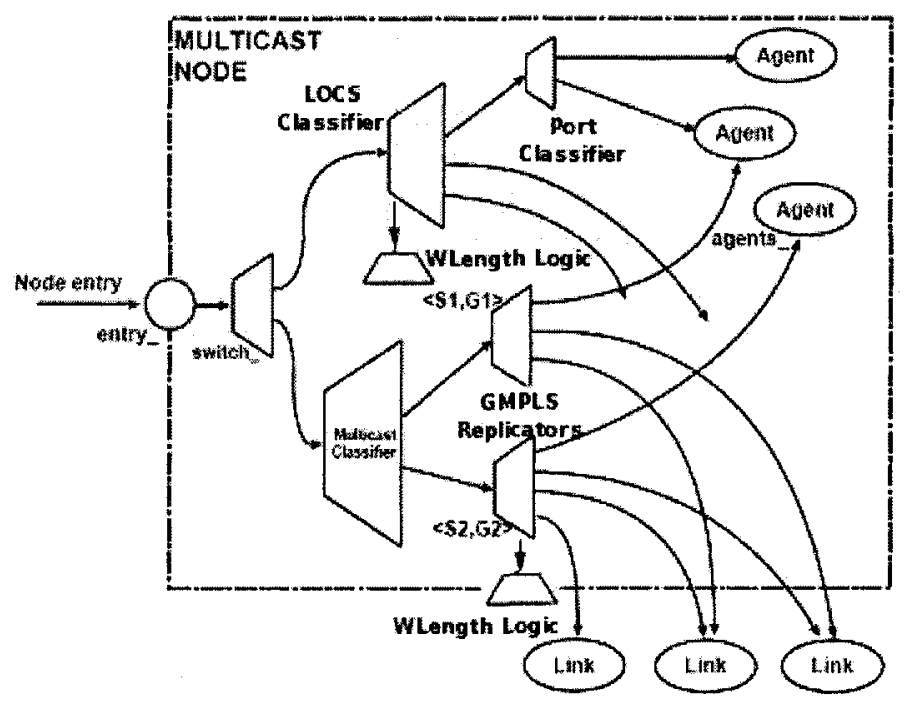

Fig. 3. NS-2 node structure developed for simulations

The LOCS classifier is the union of the MPLS node classifier and the WDM node classifier belonging to MNS and OWNS respectively. The classifier is responsible for forwarding the packets to the appropriate simulation object, such as an agent that simulates a transport protocol. In addition to this new classifier, we have built a structure (Wavelength Logic) that keeps the information on the wavelengths allocated in each link in accordance with the established LSPs, using for this purpose the MPLS Label Information Base (LIB).

In the part of the node that deals with multicast communication we have added a new replicator, the GMPLS replicator, that produces several copies of incoming information and sends the copies to its outgoing interfaces. The replicator object is essential to implement the multicast communication in NS-2.

The actions taken by this node when receiving a data packet are described below:

1. When a packet arrives at the node entry point, it is analyzed to check whether or not it is addressed to a multicast address. 
2. If it is a multicast packet, then it is forwarded to the Multicast Classifier, that, in its turn, sends the packet to the appropriate GMPLS replicator. Otherwise, the information is sent to LOCS Classifier.

3. If the next hop IP address is of this node, then the packet is passed on to the Port Classifier, to determine which agent will be responsible for handling the arriving information.

4. Otherwise, the packet is sent to the next node through the optical links using label switching.

The label switching mechanisms in the new node works as illustrated in Fig. 4.

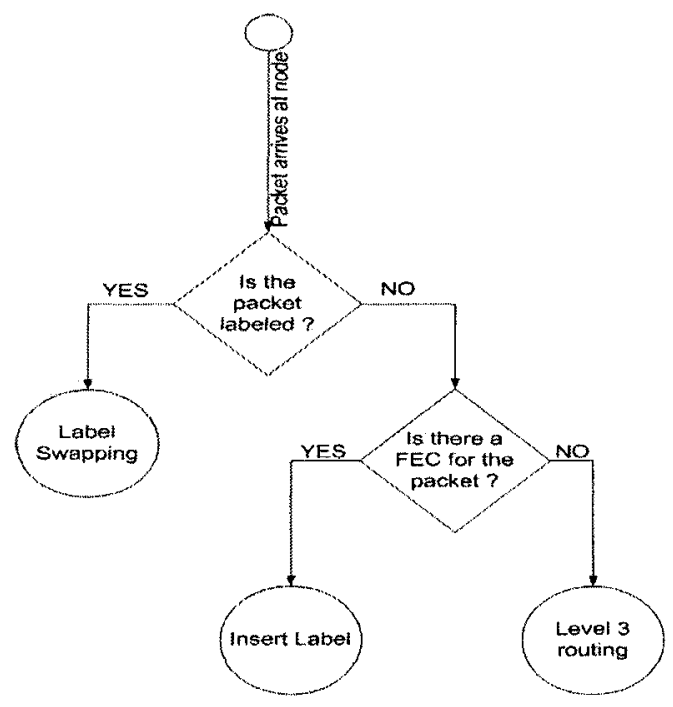

Fig 4. Label switching on the developed node

1. When a packet arrives at the LOCS classifier, it is analyzed to verify if it is labeled or not.

2. If the packet is labeled, the label swapping operation is processed (the value of the incoming label may be different from the value of outgoing label) and the information is sent for the correspondent outgoing interface. In the case that the value of the incoming label is different from the value of the outgoing label, wavelength conversion is characterized. When the packet arrives at its final destination no label swapping will be carried out.

3. If the packet is not already labeled, but there exists a FEC for it (that defines a group of packets routed in the same way), a label is inserted and Wavelength Logic is updated.

4. If no FEC is defined for the packet, it is routed at level 3 in the conventional way. 
After integrating these components, we were then able to adapt the existing functionalities of MNS, such as label switching, traffic engineering, protection and restoration, and others, to the context of optical networks provided by OWNS.

Finally, the proposed LSP triggering algorithm was implemented and incorporated in the LDP protocol of MNS and associated with the event of creation of entries in the MFC.

\subsection{A Case Study}

In this section, we present a case study to illustrate the proposed LSP triggering scheme in a possible multicast backbone in Brazil interconnecting the metropolitan networks of the state capitals of the Amazon Region with the national capital of Brasilia, using optical fibers. This network could be implemented through OCTL (Optical Cables in Transmission Lines) cables that use the OPGW technology [6].

This proposed backbone is represented in Fig. 5 and will be used in the simulations.

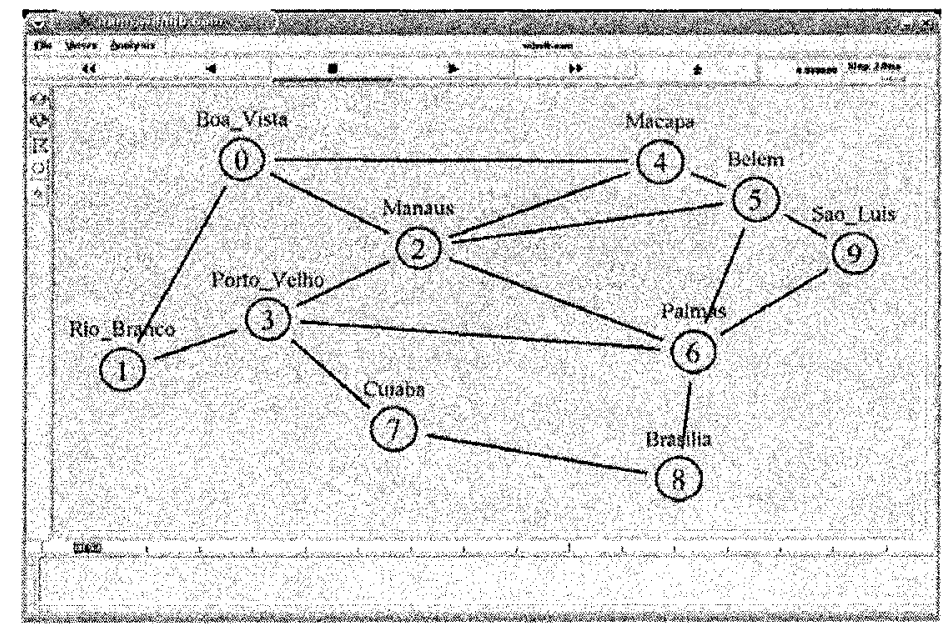

Fig. 5. Topology used in simulations

The network has 10 nodes, each one representing a capital city. In the simulations, $10 \mathrm{Gbps}$ optical links were assumed. Their propagation delays in milliseconds are listed. These delays are based in real distances. 


$\begin{array}{lll}\text { Boa Vista } & \text { Rio Branco } & 11 \mathrm{~ms} \\ \text { Boa Vista } & \text { Manaus } & 4 \mathrm{~ms} \\ \text { Boa Vista } & \text { Macapá } & 30 \mathrm{~ms} \\ \text { Rio Branco } & \text { Porto Velho } & 3 \mathrm{~ms} \\ \text { Manaus } & \text { Porto Velho } & 5 \mathrm{~ms} \\ \text { Manaus } & \text { Macapá } & 25 \mathrm{~ms} \\ \text { Manaus } & \text { Belém } & 26 \mathrm{~ms} \\ \text { Manaus } & \text { Palmas } & 20 \mathrm{~ms} \\ \text { Porto Velho } & \text { Cuiabá } & 7 \mathrm{~ms} \\ \text { Porto Velho } & \text { Palmas } & 16 \mathrm{~ms} \\ \text { Macapá } & \text { Belém } & 2 \mathrm{~ms} \\ \text { Belém } & \text { Palmas } & 6 \mathrm{~ms} \\ \text { Belém } & \text { São Luís } & 4 \mathrm{~ms} \\ \text { Palmas } & \text { São Luís } & 7 \mathrm{~ms} \\ \text { Palmas } & \text { Brasília } & 5 \mathrm{~ms} \\ \text { Cuiabá } & \text { Brasília } & 6 \mathrm{~ms}\end{array}$

The traffic type used is CBR (Constant Bit Rate) with a $4 \mathrm{Mb}$ rate. This rate can be associated with multimedia transmission in the MPEG-2 format. It was defined that the node in Brasília (Node 8 ) sends data for a multicast group composed by Belém (Node 5), Boa Vista (Node 0) and São Luís (Node 9) nodes. The multicast routing protocol used is PIM-SM (in NS-2 this protocol is known as centralized multicast) with node 6 (Palmas) as the rendezvous point. The choice for PIM-SM is due to the low density of the multicast group defined. This does not interfere in our analysis.

\subsection{Results}

We present below some of the LIBs generated by the use of the proposed LSP triggering algorithm based on the scenario described in the previous section. The purpose is to illustrate the functioning of the proposed algorithm for LSP setup. 

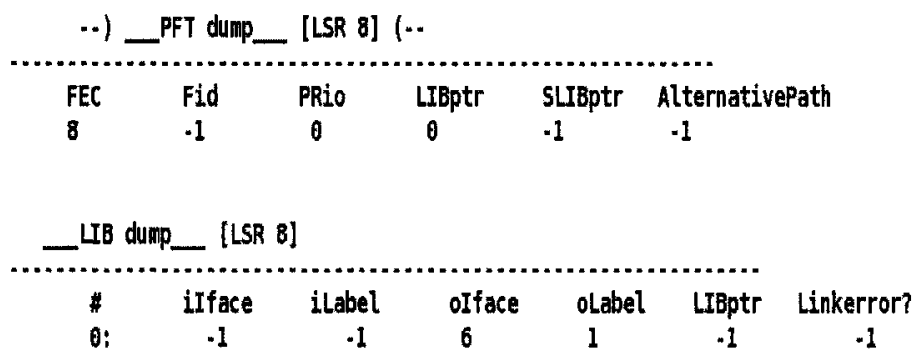

Fig 6. PFT and LIB for Node 8 (source node)

Our simulation platform inherits from the MNS module a structure known as PFT (Partial Forwarding Table), that is used to map FECs to LIB entries. The FEC value 8 refers to all multicast packets that have this node as source. If there are others traffic sources, additional LIB entries must be created at the nodes that receive or forward packets from these sources.

\begin{tabular}{|c|c|c|c|c|c|}
\hline FEC & Fid & PRio & LIBptr & SLIBptr & AlternativePa \\
\hline 8 & .1 & 0 & 0 & -1 & .1 \\
\hline 8 & .1 & 0 & 1 & .1 & .1 \\
\hline 8 & -1 & 0 & 2 & .1 & -1 \\
\hline
\end{tabular}

\begin{tabular}{|c|c|c|c|c|c|c|}
\hline \# & iIface & iLabel & oIface & OLabel & LBptr & Linkerror? \\
\hline $0:$ & 8 & 1 & 2 & 1 & .1 & .1 \\
\hline 1: & 8 & 1 & 5 & 1 & -1 & .1 \\
\hline $2:$ & 8 & 1 & 9 & 1 & .1 & .1 \\
\hline
\end{tabular}

Fig. 7. PFT and LIB for Node 6 (intermediate node)

Node 6 replicates packets, hence, we observe the creation of three LIB entries for the same FEC. Entry 0 is related to the traffic destinated to Node 0 through outgoing interface number 2. Entry 1 was created to send packets to Node 5 and Entry 2 to the traffic destinated to Node 9 . All these entries have the same incoming label. The outgoing labels may or not be the same. 


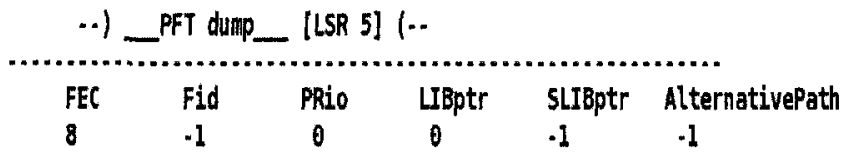

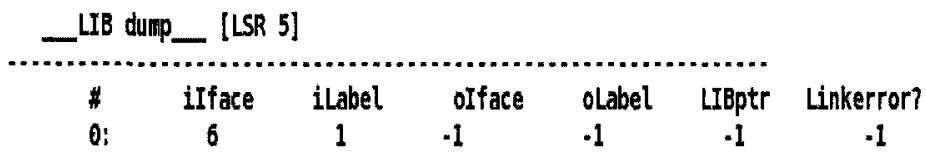

Fig. 8. PFT and LIB for Node 5 (leaf-node)

Node 5 is a destination of this multicast group. It is from this node and the other leaf nodes of this multicast group that the proposed LSP triggering scheme begins. From the LIBs generated it can be concluded that this part of LSP referring to FEC 8 (group that receives packets originated from Node 8) corresponds to the path composed by Nodes 8,6 and 5, successively. The remaining parts of the LSP are built starting at Nodes 0 and 9 , the other destinations of the group.

\subsection{Considerations about the proposed algorithm}

To initiate this LSP triggering process, traffic has to arrive at a leaf node belonging to a multicast group. This data forwarding demands level 3 routing, since there is still no LSP to route these packets at level 2, therefore, the devices used in this approach must support this functionality.

Some problems can occur in the process of creating an LSP, for instance, a label request cannot be satisfied. In this case, we need to send a signaling message from the node where the problem occurred to the leaf-nodes that triggered the LSP. This message must contain the information about the FEC related to the LSP that could not be established in order to remove the LIB entries created for this FEC. Fig. 9 illustrates this feature.

The scheme proposed here is similar to unsolicited downstream label distribution [2], with the added advantage of eliminating the signaling delay imposed when the LSP triggering is initiated at the source node, without threatening the integrity of already established LSPs, or unnecessary resource allocation, since the LSP is triggered only if there is traffic in the multicast tree. 


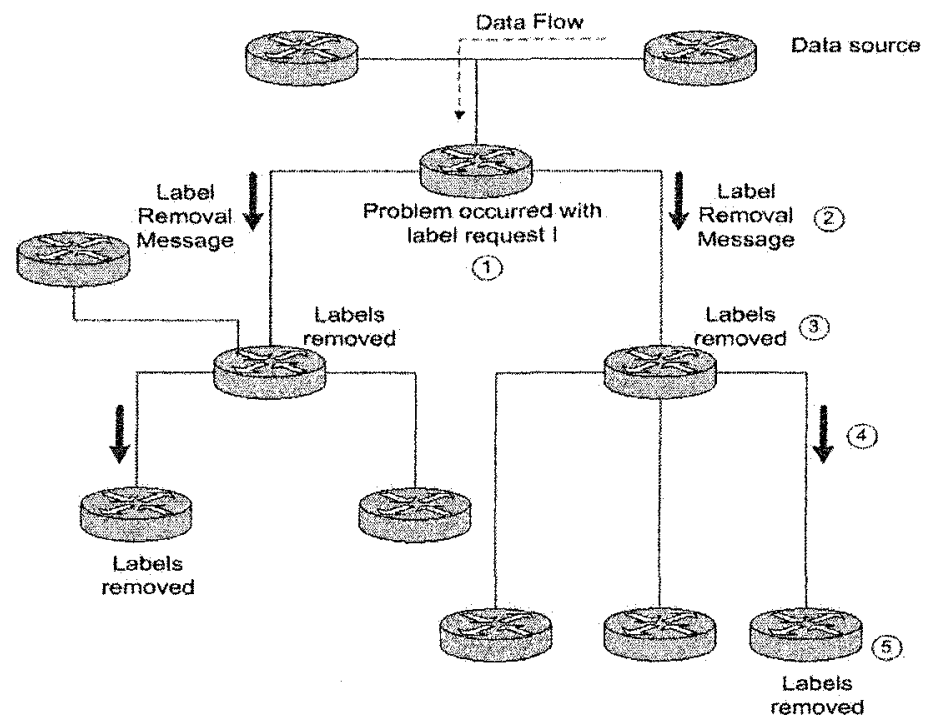

Fig. 9. Procedure in case of problems with label request.

\section{Conclusion and Future Work}

Several issues regarding to the adaptation of label switching to multicast communication in optical networks are currently being discussed and defined. This paper presents a concrete proposal for the establishment of label-switched paths using traffic-driven triggering, that is more attractive than the request-driven and topology-driven approaches, for reasons of independence of the multicast routing protocol used and lower label consumption.

The scheme proposed here is similar to unsolicited downstream label distribution [2], with the added advantage of eliminating the signaling delay imposed when the LSP triggering is initiated at the source node, without threatening the integrity of already established LSPs, or unnecessary resource allocation, since the LSP is triggered only if there is traffic in the multicast tree.

Simulations presented here show that the proposed algorithm can be implemented and satisfactorily perform the task of LSP creation. We can also see that the algorithm can minimize the blocking probability of connections, since wavelength assignment occurs in a controlled manner from the leaf nodes towards the source, and because the number of wavelength requests is small in a trafficdriven approach. Thus the probability of a wavelength being already allocated decreases.

Future studies will deal with the detailed evaluation of the impact caused by the proposed LSP triggering algorithm on the connection blocking probability, compared with other approaches, and with the analysis of traffic-engineering mechanisms in multicast communication in optical networks. 


\section{Ackowledgments}

The authors thank the finnacial support of FINEP/RNP and Eletronorte.

\section{References}

1. Abelém, A; Stanton, M. A. IP Internets Based on Optical Networks (Inter-Redes IP Baseadas em Redes Ópticas). Minicourses text book, $20^{\circ}$ Simpósio Brasileiro de Redes de Computadores (SBRC2002), Cap. 2, pp. 63-123, Búzios, RJ, Brazil. May, 2002. (in Portuguese)

2. Rosen, E. et al. Multiprotocol Label Switching Architecture. RFC3031. January, 2001.

3. Mannie E. (Editor). Generalized Multi-Protocol Label Switching (GMPLS) Architecture. Internet Draft, draft-ietf-ccamp-gmpls-architecture-04.txt. February, 2003.

4. Ooms, D. et al. Framework for IP Multicast in MPLS. RFC 3353. August, 2002.

5. Ooms, D. et al. MPLS for PIM-SM. Internet Draft, draft-ooms-mpls-pimsm-00.txt. November, 1998.

6. Murthy, C.; Gurusamy, M. WDM Optical Networks: Concepts, Design, and Algorithms, Prentice Hall PTR, Nov. 2001.

7. Rajagopalan, B. et al. IP over Optical Networks: A Framework. RFC3717. March, 2004.

8. Magalhães, M.; Cardozo, E. Introduction to IP Label-switching through MPLS (Introdução à comutação IP por rótulos através de MPLS). Minicourses text book, $19^{\circ}$ Simpósio Brasileiro de Redes de Computadores (SBRC2002), Cap. 3, Florianópolis, SC, Brazil. May, 2001. (in Portuguese)

9. Fall, K.; Varadhan, V. The $n s$ Manual. Url: http://www.isi.edu/nsnam/ns . Accessed in: July, 2005.

10. Ahn, G; Chun, W. Design and Implementation of MPLS Network Simulator. Url: http://flower.ce.cnu.ac.kr/ fogl/mns/. Accessed in: July, 2005.

11. Wen, B; Bhide, N. M.; Shenai, R. K.; Sivalingam, K. M.Optical Wavelength Division Multiplexing (WDM) Network Simulator (OWns): Architecture and Performance Studies. In: SPIE Optical Networks Magazine Special Issue on Simulation, CAD, and Measurement of Optical Networks, March, 2001.

12. Esteves, R.; Nagahama, F; Abelém, A.; Stanton, M. A Proposal to Adjust the GMPLS Control and Signaling Mechanisms for Optical Burst Switched Networks. In: Annals of 3rd International Information and Telecommunication Technologies Symposium (I2TS2004). São Carlos, SP, Brazil. December, 2004.

13. Viana, J.; Esteves, R; Abelém, A; Costa, J. C.; Stanton, M. Analysis of Failure-Recovery Mechanisms in Next-Generation Optical Networks with Control Plane Based on GMPLS (Análise de Mecanismos de Recuperação de Falhas em Redes Ópticas de Nova Geração com Plano de Controle Baseado no GMPLS). In: IV Workshop em Desempenho de Sistemas Computacionais e de Comunicação-WPERFORMANCE (SBC2005), São Leopoldo, RS, Brazil. July, 2005. (in Portuguese)

14. Boudani, A.; Cousin, B. Multicast Routing Simulator over MPLS Networks. 36th Annual Simulation Symposium, Orlando, Florida, USA, March 2003. 